\title{
Association of treatment adherence with real-life VA outcomes in AMD, DME, and BRVO patients
}

This article was published in the following Dove Press journal:

Clinical Ophthalmology

\author{
Christoph Ehlken ${ }^{1,2}$ \\ Mandy Helms' \\ Daniel Böhringer' \\ Hansjürgen T Agostini' \\ Andreas Stahl' \\ 'Ophthalmology, Eye Center, \\ Medical Center, Faculty of Medicine, \\ University of Freiburg, Freiburg \\ im Breisgau, ${ }^{2}$ Ophthalmology, \\ Eye Center, University Hospital \\ Schleswig-Holstein, Campus \\ Kiel, Germany
}

Purpose: Real-life clinical outcomes of patients treated with anti-VEGF drugs for neovascular age-related macular degeneration (nAMD), diabetic macular edema (DME), or macular edema secondary to branch retinal vein occlusion (BRVO) are often inferior to results from randomized clinical trials. This observational cohort study investigates treatment adherence and real-life clinical outcomes within the first year of treatment.

Patients and methods: A total of 708 treatment-naïve patients (466 nAMD, 134 DME, and 108 BRVO) were included. Patients were followed with a PRN treatment protocol with three intravitreal injections (IVIs) and a series of 3 monthly injections in case of persistent or recurrent disease activity, as determined by monthly follow-up exams including optical coherence tomographies. Occurrence of gaps of $>56$ days between treatments or follow-up (nonadherence [NA]) and the reasons for NA (patient- or center-associated) as well as disease activity within the first 12 months of treatment were analyzed. Visual acuity (VA) as well as numbers and dates of optical coherence tomography and IVI were extracted from medical records.

Results: NA occurred significantly more often in patients with DME (44\%) than nAMD $(32 \%)$ or BRVO $(25 \%, p<0.01$ between groups). NA was mainly patient-associated (nAMD: 80.0\%, DME: $83.1 \%$, BRVO: $70.4 \%, p=0.38$ between groups). Patients with nAMD and DME and appropriate treatment/follow-up adherence had a better chance of significantly gaining or maintaining VA, respectively (19.9\% vs $12.0 \%$ with 3-line-gain in nAMD and $1.3 \%$ vs $15.3 \%$ 3 -line loss in DME; each $p<0.05$ ). NA did not correlate with VA outcomes in BRVO (3-line gain $30.9 \%$ vs $48.1 \%$ and 3 -line loss $8.6 \%$ vs $7.4 \%$; $p>0.05$ ).

Conclusion: NA to treatment and follow-up regimens is a common problem in the management of patients with AMD and DME and limits clinical treatment outcomes under real-life conditions. Patients with DME have the highest risk of patient-associated NA, associated with a higher risk for significant VA loss.

Keywords: AMD, RVO, DME, adherence, anti-VEGF, PRN, follow-up

\section{Introduction}

The efficacy of anti-VEGF treatment with ranibizumab, bevacizumab, or aflibercept has been proven in several Phase III randomized clinical trials for neovascular age-related macular degeneration (nAMD), ${ }^{1,2}$ diabetic macular edema (DME), ${ }^{3,4}$ and branch retinal vein occlusion (BRVO). ${ }^{5-7}$ Phase IV trials, however, found inferior clinical outcomes under real-life conditions compared with controlled treatment trials. ${ }^{8-10}$

In AMD, Phase III trials found a significant gain in best-corrected visual acuity (BCVA) after treatment that could be maintained over a course of 24 months., ${ }^{1,2,11}$ Real-life studies, however, found only a stabilization of BCVA and the initial VA gain was lost over time in most patients. A subgroup analysis of the AURA study revealed 
that differences in the management of patients with nAMD in different countries (eg, in regard to the number of intravitreal injections (IVIs) administered, or the number of examinations and optical coherence tomography (OCT) performed) were associated with differences in clinical outcomes. ${ }^{9}$ There was a tendency toward better clinical outcomes in countries where more control examinations, OCTs, and IVI were performed. In DME, VA outcomes might be comparable between Phase III studies and clinical reality with a higher proportion of patients keeping their initial BCVA gains. ${ }^{12-14}$ However, most of this data are derived from extensions of Phase III trials and might not correctly represent true clinical reality. For BRVO, there is only little evidence available on long-term real-life outcomes.

Identifying the factors that contribute to inferior treatment outcomes in clinical real-life settings is important to deliver best possible care to IVI patients. A longer distance between the patients' home and their treatment center, subjective dissatisfaction with treatment results, the burden of frequent follow-up visits, high age, low BCVA at baseline, or poor patient knowledge have been associated with reduced treatment outcomes in nAMD, ${ }^{15,16}$ probably by promoting nonadherence (NA). NA can be defined as a deviation from treatment recommendations (either by the patient or by the treating physician/center). There are only limited data on NA in macular diseases other than AMD. In addition, there are no studies distinguishing between center- and patient-associated NA.

In this study, we analyze the extent and impact of NA in $\mathrm{nAMD}$, but also in DME and BRVO, and we seek to differentiate between patient- and center-related reasons.

\section{Patients and methods}

\section{Study design}

All patients in this retrospective, observational cohort study were treated at the Eye Center, University of Freiburg, Germany, according to the treatment recommendations of the German Ophthalmic Society and the German Retina Society (available at http://www.dog.org/?cat=7\#9). ${ }^{17,18}$ The study was registered in the German Clinical Trials Register(DRKS) as DRKS00007999. Ethical approval was obtained from the institutional review board of the University of Freiburg, Germany. Patients signed written informed consent prior to inclusion in the study.

At the study center (Eye Center, University Hospital, Freiburg), the routine anti-VEGF treatment protocol was comparable to the IVAN PRN scheme. For patients with nAMD, DME, or BRVO, the protocol stipulates an upload of 3 monthly IVIs of ranibizumab, aflibercept, or bevacizumab, followed by OCT and fundus exam 4 weeks later. In case of persistent or recurrent disease activity, one to three additional
4- to 6-weekly IVIs are scheduled, depending on disease activity. In cases with no signs of disease activity, monthly follow-up visits with BCVA and spectral domain-OCT are performed for up to 6 months after the last IVI.

\section{Study objectives}

The study was conceived to analyze the extent and impact of NA on clinical results in the treatment of the nAMD, DME, and BRVO. The main predefined end points of this 12-monthstudy were: proportion of patients with significant $\mathrm{VA}$ gain or loss ( $\geq 3$ BCVA lines), change of VA, extent of NA (defined as unintended treatment/examination gaps $>56$ days) and reasons for NA, and differences between indications.

\section{Study collective and inclusion/exclusion criteria}

Eligible patients were identified via a search in the institutional scheduling database for all patients who were started on anti-VEGF treatment for nAMD, DME, and BRVO between November 2011 and March 2014 at the Eye Center, University Hospital, Freiburg, Germany. Exclusion criteria were IVIs in the context of an interventional clinical study, IVIs for medication other than anti-VEGF (eg, steroids), and any other intraocular surgery within the first 12 months of anti-VEGF treatment (eg, phacoemulsification). Patients with central retinal vein occlusion (CRVO) were not included, as patient numbers were expected to be too low for meaningful interpretation.

\section{Data acquisition}

Clinical data, including disease group, dates of injections and OCTs, and VA before first treatment and after $12 \pm 2$ months (or at last examination before 12 months), were electronically extracted from clinical records and manually reviewed. Lastobservation-carried forward analysis was used if VA was not available at $12 \pm 2$ months. Only one eye per patient was chosen as study eye. If both eyes fulfilled the aforementioned criteria, the right eye was included.

Dates of consultations (treatments or examinations with OCT) were analyzed for the occurrence of gaps between treatments and/or follow-up of $>56$ days. Eight weeks (56 days) was chosen as a cutoff to respect the intended interval of 4 weeks (28 days) between follow-ups or treatments on the one hand, and to allow for individual variations (maximally +4 weeks, for example, in case of rescheduled or missed visits) on the other hand. Patients without any gap $>56$ days were classified as "continuously adherent." If the treating physician considered further treatment or examinations unnecessary, the patient was classified as 
“treatment success" or "intended gap." Unintended gaps $>56$ days between treatments led to the classification "nonadherence" (NA). If more than one gap $>56$ days occurred within the first 12 months of treatment, only the first gap was analyzed.

NA was manually coded whether it was caused by the patient (did not show up for treatment/follow-up) or the treatment center (no/late appointment scheduled) and whether the underlying disease was active (treatment recommended) or inactive (follow-up recommended) at the last visit before the gap occurred.

\section{Statistical analysis}

Groups were analyzed using descriptive statistics. Data are presented as mean and standard deviation (SD) for continuous variables and patient counts and percentages for categorical variables. Data were compared between diseases and follow-up subgroups (adherent vs nonadherent), using the two-sided Wilcoxon's test for independent samples (between groups) or dependent samples (within groups) for continuous variables and the Fisher's exact test for categorical variables, where appropriate. VA was transformed to $\log$ MAR before analysis.

Kaplan-Meier-analysis was performed to estimate the incidence of NA. Log-rank test was used to analyze for differences in time to NA between groups (all NA, patientassociated NA).

Logistic regression was performed to identify factors associated with patient-associated NA (occurrence of NA as dependent variable) in the nAMD, DME, and BRVO subgroups.

$P$-values $<0.05$ were considered statistically significant. Because of the exploratory nature of the study, no correction for multiple testing was performed. Statistics were performed using the freely available statistic software R (version 3.3.1) including Rcmdr packages. ${ }^{19}$

\section{Results}

\section{Study group and adherence}

A total of 1,269 patients signed informed consent. Of those, 561 were excluded (389 indication not suitable, 148 receiving co-treatment, 16 participating in other clinical studies, and 8 receiving treatment elsewhere). Consequently, 708 patients were included in the study. Of those, 466 were treated for nAMD, 134 for DME, and 108 for BRVO.

Distribution of treatment success and continuous follow-up and NA for nAMD, DME, and BRVO are shown in Figure 1. In case of persistent or recurrent disease activity, the median turnaround time from indication to IVI treatment was 21 days.

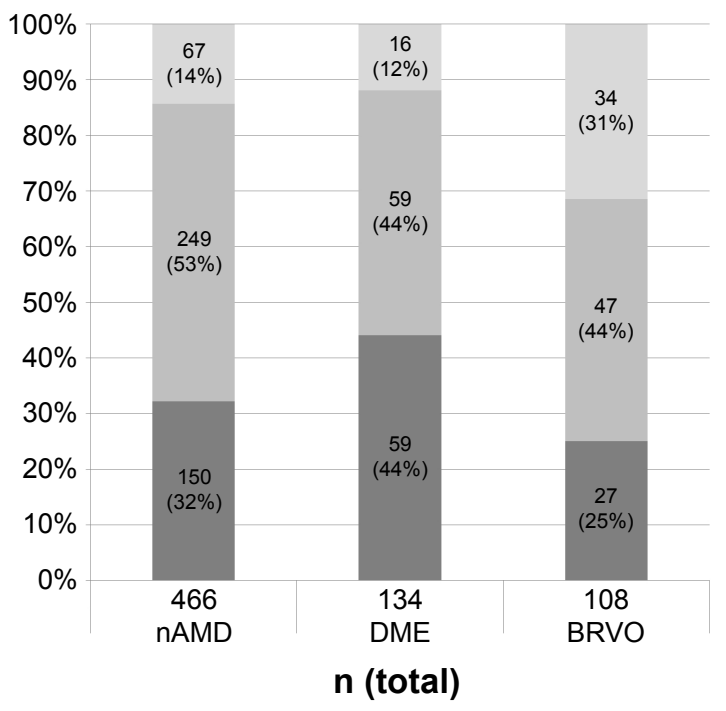

Success $\square$ Continuous adherence $\square$ Nonadherence

Figure I Follow-up over 12 months in nAMD, DME, and BRVO patients. Notes: Success, no treatment or follow-up necessary, leading to intended gap $>56$ days; continuous adherence, patient underwent frequent treatments or follow-ups at least every 56 days; nonadherence, at least one unintended gap $>56$ days between treatments or follow-ups; this subgroup is further analyzed in Figure 2. Abbreviations: nAMD, neovascular age-related macular degeneration; DME, diabetic macular edema; BRVO, branch retinal vein occlusion.

Treatment success (leading to intended gaps $>56$ days between consultations) was seen in 67 of 466 nAMD patients (14.4\%), 16 of 134 DME patients (11.9\%), and 34 of 108 BRVO patients ( $31.5 \%$; $p \leq 0.001$ between groups). Continuous treatment or follow-ups were performed in $53.4 \%$ of nAMD patients, $44.0 \%$ of DME patients, and $43.5 \%$ of BRVO patients $(p=0.053)$. Consequently, only every second DME patient in our study was treated in line with the intended follow-up regimen $(67.8 \%$ in nAMD, $56.0 \%$ in DME, and $75.0 \%$ in BRVO, $p<0.01$ between groups). NA was more common in the DME group (44.0\%) than in nAMD $(32.2 \%)$ or BRVO $(25.0 \%, p<0.01$ between groups $)$.

The reasons for NA were further analyzed and are shown in Figure 2. In most cases, NA was patient-associated, regardless of the underlying disease (nAMD $80.0 \%$ patientassociated vs $20.0 \%$ center-associated, DME $83.1 \%$ vs $26.9 \%$, and BRVO 70.4\% vs 29.6\%; $p=0.38$ between groups of nonadherent patients). Disease activity was present in almost every second patient with DME but only every third patient with BRVO at the time when the first gap $>56$ days occurred $(40.0 \%$ in nAMD, $47.5 \%$ in DME, and $33.3 \%$ in $\mathrm{BRVO} ; p=0.30$ between groups). In addition, disease activity was more common in patient-associated NA than in centerassociated NA (44.1\% vs $27.1 \%, p=0.033$ ).

Relating to the total number of included patients (adherent and nonadherent), patient-associated NA was more common 


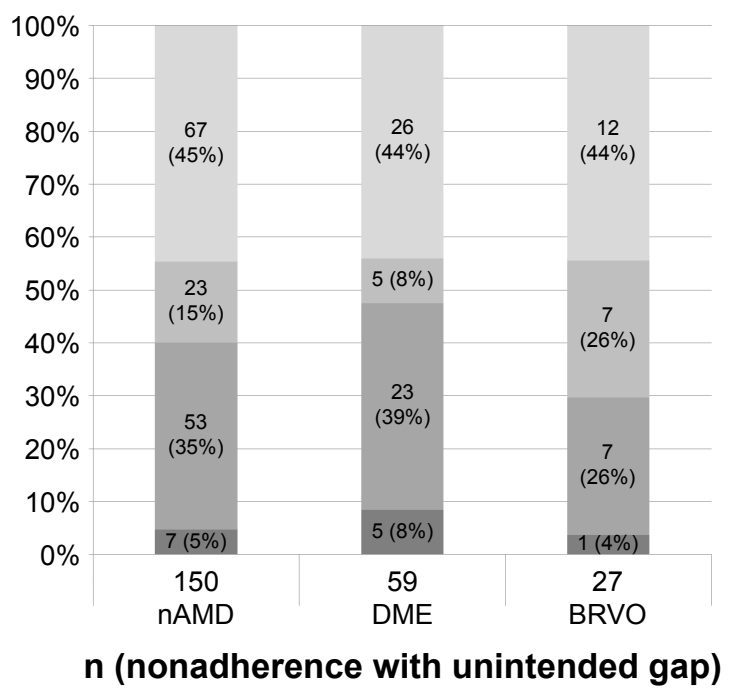

Patient/inactive $\quad$ Center/inactive
Patient/active $\quad$ Center/active

Figure 2 Reasons for unintended treatment gaps (NA).

Notes: Patient, patient-associated NA; center, center-associated NA; inactive, no treatment necessary at last visit before NA; active, treatment intended at last visit before NA.

Abbreviations: nAMD, neovascular age-related macular degeneration; DME, diabetic macular edema; BRVO, branch retinal vein occlusion; NA, nonadherence.

in DME than in nAMD or BRVO (nAMD: $25.8 \%$, DME: $36.6 \%$, and BRVO: $17.6 \% ; p<0.01$ ). Center-associated NA was comparable between groups (nAMD: 6.4\%, DME: 7.5\%, and BRVO: $7.4 \% ; p=0.84$ ).

Kaplan-Meier estimation shows a continuous decline of adherence in all disease groups, with a steeper decline in DME patients compared with nAMD and BRVO $(p<0.01$ between groups, all patients, log-rank test, and Kaplan-Meier estimation, Figure 3).

The median duration of treatment gaps was 79.5 days (mean \pm SD: $144.4 \pm 161.1$ days) in $n A M D, 88.0$ days (164.9 \pm 167.5$)$ in DME, and 72.0 days $(98.0 \pm 56.5)$ in BRVO. The proportion of nonadherent patients who did not return at all for further treatments or examinations (loss to follow-up) was significantly different between diseases: while $93.7 \%$ of nonadherent patients with BRVO returned for further follow-up, only two-thirds of patients with nAMD and DME returned for further appointments (69.9\% and 69.5\%, respectively, $p<0.001$ between all groups).

\section{Factors associated with patient- associated NA}

Odds ratios (ORs) for all tested factors are shown in Table 1. In the nAMD subset, higher age (OR: 1.04, $p=0.013)$ and poor VA at baseline (OR: $2.37, p=0.048$ ) were statistically significantly associated with higher risk for patient-associated

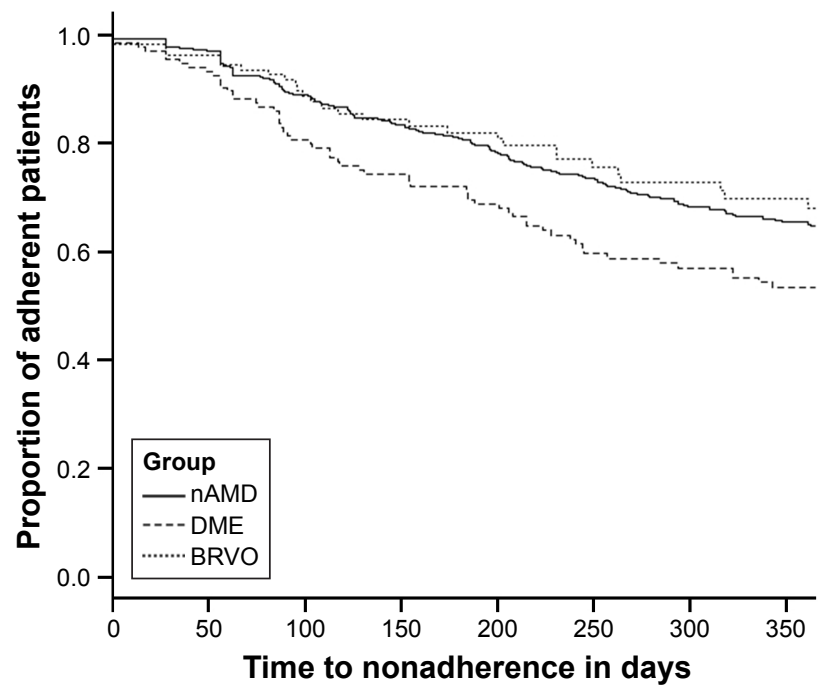

Number at risk

$\begin{array}{llllllll}\text { nAMD 466 } & 451 & 389 & 355 & 331 & 295 & 259 & 241 \\ \text { DME 134 } & 125 & 104 & 94 & 87 & 68 & 64 & 59 \\ \text { BRVO 108 } & 104 & 86 & 75 & 69 & 55 & 49 & 43\end{array}$

Figure 3 Kaplan-Meier estimation of incidence of NA over the course of 365 days. Note: $p=0.014$ between groups, log-rank test.

Abbreviations: NA, nonadherence; nAMD, neovascular age-related macular degeneration; DME, diabetic macular edema; BRVO, branch retinal vein occlusion.

NA. In the DME and BRVO subsets, no statistically significant associations were identified.

\section{Association of NA with clinical outcomes}

Patients with continuous follow-up or treatment success (ie, intended gaps in line with treatment protocol) had more IVI and OCT scans than nonadherent patients in all disease entities (Table 2). In nAMD and DME, both IVIs and OCTs were performed more often in the subgroup with good treatment adherence. Association of adherence with VA outcomes is shown in Figure 4. A higher proportion of adherent nAMD patients improved by at least 3 BCVA lines, compared with nonadherent nAMD patients ( $19.9 \%$ vs $12.0 \%$, respectively, $p=0.035$ ), while the percentage with a loss of at least three lines was comparable. In DME, a gain of at least three lines was comparable between adherent and nonadherent subgroups. However, significantly more nonadherent DME patients experienced clinically significant VA loss $(1.3 \%$ vs $15.3 \%, p<0.01)$. We observed no statistically significant differences between adherent and nonadherent BRVO subgroups regarding VA development.

\section{Discussion}

In this study, NA, defined as unintended gaps $>56$ days between treatments/follow-ups, occurred in a significant number of patients (nAMD: 32\%, DME: 44\%, and BRVO: 
Table I Regression analysis for risk factors for patient-associated NA

\begin{tabular}{|c|c|c|c|c|c|c|}
\hline \multirow[t]{2}{*}{ Variable } & \multicolumn{2}{|l|}{ nAMD } & \multicolumn{2}{|l|}{ DME } & \multicolumn{2}{|l|}{ BRVO } \\
\hline & OR $(95 \% \mathrm{Cl})$ & $p$-value & OR $(95 \% \mathrm{Cl})$ & $p$-value & OR $(95 \% \mathrm{Cl})$ & $p$-value \\
\hline Intercept & $0.01(0.00 ; 0.13)$ & $<0.001$ & $1.79(0.10 ; 30.8)$ & 0.689 & $0.26(0.00 ; 34.0)$ & 0.588 \\
\hline Age (years) & 1.04 (I.0I; I.06) & 0.013 & $0.99(0.96 ; 1.03)$ & 0.757 & $1.00(0.94 ; 1.05)$ & 0.876 \\
\hline Sex (female) & $0.74(0.48 ; 1.16)$ & 0.189 & $0.57(0.25 ; 1.31)$ & 0.185 & $\mathrm{I} .59(0.5 \mathrm{I} ; 4.97)$ & 0.426 \\
\hline Mobility impaired & $0.98(0.48 ; 2.00)$ & 0.961 & $\mathrm{I} .27(0.34 ; 4.79)$ & 0.720 & $0.63(0.07 ; 6.00)$ & 0.686 \\
\hline Upload successful & $\mathrm{I} .07(0.4 \mathrm{I} ; 2.82)$ & 0.892 & $0.30(0.08 ; 1.23)$ & 0.094 & $0.72(0.06 ; 9.25)$ & $0.80 \mathrm{I}$ \\
\hline VA study eye $>0.1-0.3 \log M A R^{a}$ & $2.42(0.90 ; 6.49)$ & 0.079 & $1.37(0.40 ; 4.75)$ & 0.617 & $0.38(0.02 ; 7.50)$ & 0.525 \\
\hline VA study eye $>0.3$ logMAR ${ }^{a}$ & $2.37(1.01 ; 5.57)$ & 0.048 & $1.75(0.54 ; 5.73)$ & 0.352 & $2.0(0.21 ; 19.3)$ & 0.550 \\
\hline Study eye better than fellow eye & $0.93(0.60 ; 1.46)$ & 0.765 & $\mathrm{I} .58(0.7 \mathrm{I} ; 3.5 \mathrm{I})$ & 0.259 & $0.46(0.14 ; 1.57)$ & 0.214 \\
\hline Both eyes treated & $1.23(0.67 ; 2.26)$ & 0.503 & $0.52(0.20 ; 1.36)$ & 0.185 & $<0.00(0 ;$ inf $)$ & 0.993 \\
\hline
\end{tabular}

Notes: aCompared with VA study eye $<0.1$ logMAR. Statistically significant $p$-values $(<0.05)$ are shown in bold.

Abbreviations: NA, nonadherence; nAMD, neovascular age-related macular degeneration; DME, diabetic macular edema; BRVO, branch retinal vein occlusion; OR, odds ratio; $\mathrm{Cl}$, confidence interval; $\mathrm{VA}$, visual acuity.

$25 \%$ ) and was associated with inferior VA outcomes in nAMD and DME.

Compared with other current literature, NA in nAMD patients was relatively low in this analysis. The PONS study found NA in up to $95 \%$ after 12 months in patients with nAMD in Germany. An analysis of patients $>90$ years in Denmark described numbers comparable to our study (NA in approximately 50\% after 12 months). ${ }^{20}$ Larger observational studies reported unexpectedly low numbers of IVI and OCT, without providing evidence for the cause of these low numbers. ${ }^{8,9}$ The relatively low amount of NA in this analysis may be explained by the establishment of an intensified follow-up protocol with monthly examinations for up to 6 months after the last IVI treatment. In addition, the study center is the main care provider for IVI treatment in this region of Germany, and so the problems between care providers (eg, referral ophthalmologist and treating ophthalmologist) play a minor role. There is no comparable published data on NA in patients with DME and RVO.

The definition of NA is crucial, yet not standardized. Different treatment protocols, such as monthly, PRN, or treat-and-extend, may need to define NA differently. In our treatment center (Freiburg), a 4-weekly treatment/ examination PRN scheme was pursued. treat-and-extend was not used at the time of data collection. Thus, 8 weeks (56 days) was chosen as a reasonable cutoff to identify significant deviations from the intended 4-weekly treatment/ examination PRN scheme.

Several explanations for patient-associated NA have been suggested. The PONS study investigated possible factors leading to NA in nAMD patients, including clinical parameters, and demographical and socioeconomic factors (eg, education, impaired mobility, or need for help with transport to injections). ${ }^{10,16}$ However, no relevant correlation could be identified. In a study by Boulanger-Scemama et al, ${ }^{15}$ patients indicated problems with mobility or needing help to keep treatment appointments as a major hindrance especially in the long term. Further barriers were disappointment in clinical results and the need for frequent follow-up examinations. Higher age and poor BCVA at baseline, as well as a long distance to the treating center were associated with poor adherence.

In our analysis, higher age and poor baseline VA were associated with a higher risk for NA in the nAMD subgroup, but not in the DME and BRVO subgroups. These patients were younger and had better baseline BCVA compared with patients with nAMD. This suggests that factors contributing to NA may be different between diseases. An explanation may be the presence of additional comorbidities in patients with DME, since the congregation of several risk factors was shown earlier to be associated with higher risk for NA. In these cases, a patient's attention may be focused on other conditions requiring treatment or care, so that from the patient's perspective the retinal disease may be subordinated. Cooperation and intense correspondence between treating physicians from different specialties has been proven to be beneficial in promoting adherence in these patients. ${ }^{21}$ Socioeconomic factors (such as status of employment or education) might contribute as well, but could not be analyzed in this retrospective study.

The inferiority of real-life clinical outcomes compared with Phase-III trials in nAMD, defined as a lower mean gain or even loss of VA, has been described in several larger studies. ${ }^{8,9,22}$ An undersupply with IVI and OCT is most commonly used to explain this inferiority. In our study, mean VA did not differ significantly between adherent and nonadherent subgroups, although the percentage of nAMD patients gaining significantly was higher in the adherent subgroup. This can probably be explained by the relatively high number of IVI and OCT even in the nonadherent subgroup of patients, 


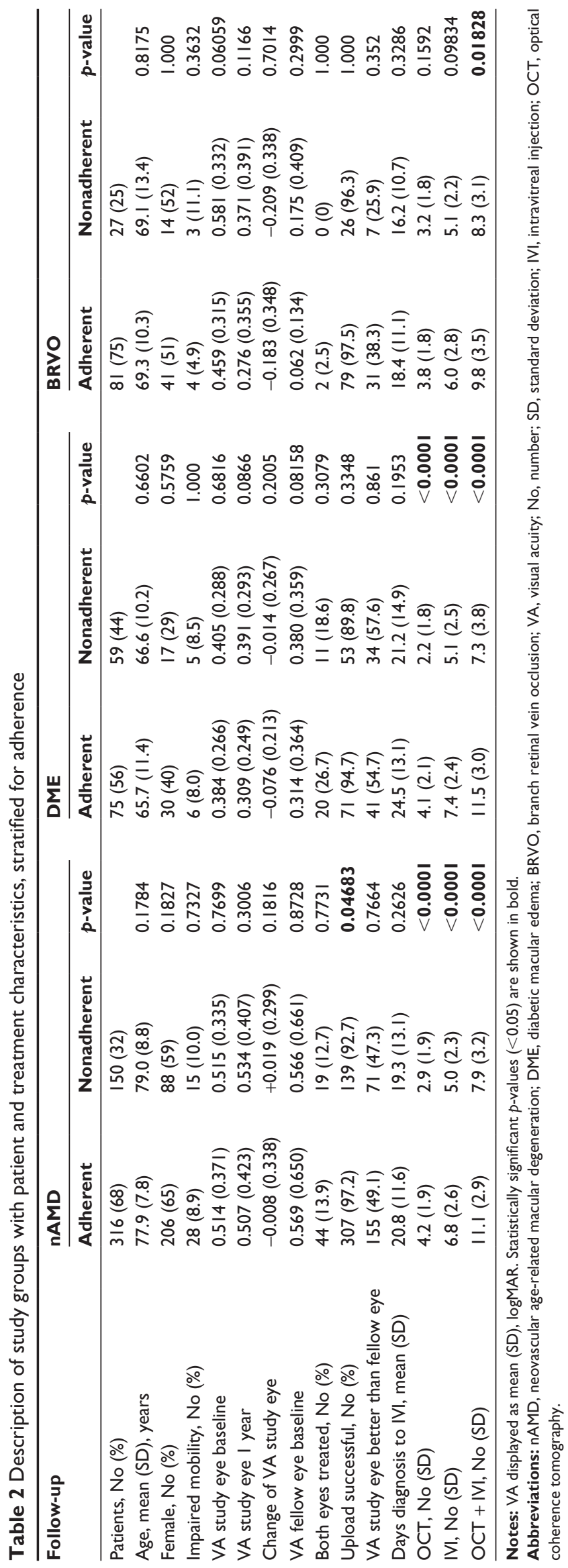

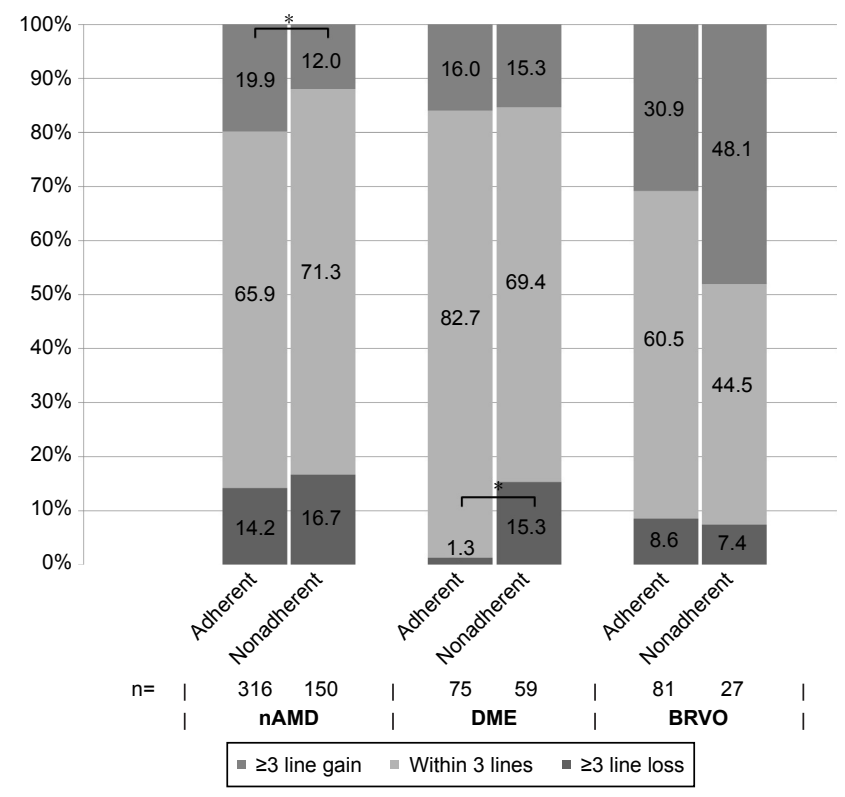

Figure 4 Development of visual acuity after 12 months for nAMD, DME, and BRVO, stratified for follow-up (adherent/nonadherent).

Note: $* p<0.05$.

Abbreviations: nAMD, neovascular age-related macular degeneration; DME, diabetic macular edema; BRVO, branch retinal vein occlusion.

which is higher than, for example, in AURA (2.9 OCT and 5.0 IVI in this study vs 2.1 and 4.3 in AURA, Germersheim, Germany). ${ }^{23}$ A study investigating an intensified monitoring program, however, has failed to prove an advantage over conventional follow-up in patients with nAMD. One shortcoming in the current literature is that most studies do not differentiate between patient- and center-associated NA. In our analysis, center-associated NA, which means that appointments for further follow-ups or injections could not be arranged in the intended timely manner, occurred in $<10 \%$ of all patients. While this percentage is lower compared with the patientassociated reasons for NA, center-associated NA may contribute to the observed real-life clinical outcomes. However, it has to be acknowledged that center-associated NA may differ between institutions, health systems, or countries, depending on the organization and reimbursement procedures.

In our study, as well in others, good long-term therapy adherence was associated with better VA outcomes. Interestingly, in nAMD, a difference was seen in the percentage of patients gaining 15 letters, while the percentages of patients with 15-letter-loss and mean VA were comparable. In DME, however, NA was correlated predominantly with the proportion of patients losing 15 letters. The reasons for these differences cannot be deduced from this study. It is, however, important to acknowledge the presence of such differences and potentially intensify patient counseling particularly for 
DME patients at risk of significantly losing VA. In our cohort, we did not find any statistically significant differences for VA outcomes in adherent vs nonadherent BRVO subgroups, which may in part be explained by the smaller number of patients but may also reflect the overall better VA prognosis.

Our study had several limitations. Because of the retrospective design, there was no mandatory treatment protocol. Treatment was, however, based on official treatment recommendations in Germany, as explained above. Some data could not be obtained retrospectively, such as reasons for patientassociated treatment gaps. In addition, VA at 12 months was not available for all patients, and a last observation carried forward strategy (VA at the least registered appointment before treatment discontinuation) was used. This may lead to a selection and a reporting bias, which cannot be completely ruled out in a retrospective analysis of NA. Strengths of the study include the ability to discriminate between center- and patient-associated causes of NA, as well as the inclusion of three disease entities (nAMD, DME, and BRVO), allowing comparison of NA in different clinical settings. The number of patients is considerably lower for DME and BRVO compared with nAMD, and thus, the study may be underpowered for some statistical evaluations particularly in these groups.

\section{Conclusion}

NA was common in nAMD, DME, and BRVO, and NA occurred more often in DME than in nAMD or BRVO. NA was predominantly patient-associated in all groups. NA was associated with inferior clinical outcomes in patients treated for $\mathrm{nAMD}$ and DME.

\section{Acknowledgment}

We thank T Post, C Geithner, and S Leidigkeit for taking excellent care of all patients in the VEGF treatment path.

\section{Author contributions}

All authors contributed toward data analysis, drafting and revising the paper and agreed to be accountable for all aspects of the work.

\section{Disclosure}

The authors report no conflicts of interest in this work.

\section{References}

1. Moja L, Lucenteforte E, Kwag KH, et al. Systemic safety of bevacizumab versus ranibizumab for neovascular age-related macular degeneration. Cochrane Database Syst Rev. 2014;(9):CD011230.

2. Sarwar S, Clearfield E, Soliman MK, et al. Aflibercept for neovascular age-related macular degeneration. Cochrane Database Syst Rev. 2016; 2:CD011346.
3. Mitchell P, Bandello F, Schmidt-Erfurth U, et al. The RESTORE study: ranibizumab monotherapy or combined with laser versus laser monotherapy for diabetic macular edema. Ophthalmology. 2011;118(4):615-625.

4. Nguyen QD, Shah SM, Khwaja AA, et al. Two-year outcomes of the ranibizumab for edema of the mAcula in diabetes (READ-2) study. Ophthalmology. 2010;117(11):2146-2151.

5. Brown DM, Campochiaro PA, Bhisitkul RB, et al. Sustained benefits from ranibizumab for macular edema following branch retinal vein occlusion: 12-month outcomes of a Phase III study. Ophthalmology. 2011;118(8):1594-1602.

6. Clark WL, Boyer DS, Heier JS, et al. Intravitreal aflibercept for macular edema following branch retinal vein occlusion: 52 -week results of the VIBRANT Study. Ophthalmology. 2016;123(2):330-336.

7. Narayanan R, Panchal B, Das T, et al. A randomised, double-masked, controlled study of the efficacy and safety of intravitreal bevacizumab versus ranibizumab in the treatment of macular oedema due to branch retinal vein occlusion: MARVEL Report No. 1. Br J Ophthalmol. 2015;99(7):954-959.

8. Finger RP, Wiedemann P, Blumhagen F, Pohl K, Holz FG. Treatment patterns, visual acuity and quality-of-life outcomes of the WAVE study - a noninterventional study of ranibizumab treatment for neovascular age-related macular degeneration in Germany. Acta Ophthalmol. 2013;91(6):540-546.

9. Holz FG, Tadayoni R, Beatty S, et al. Multi-country real-life experience of anti-vascular endothelial growth factor therapy for wet age-related macular degeneration. Br J Ophthalmol. 2015;99(2):220-226.

10. Ehlken C, Wilke T, Bauer-Steinhusen U, Agostini HT, Hasanbasic Z, Müller S. Treatment of neovascular age-related macular degeneration patients with vascular endothelial growth factor inhibitors in everyday practice: Identification of Health Care Constraints in Germany-The PONS Study. Retina. Epub 2017 May 9.

11. Schmidt-Erfurth U, Kaiser PK, Korobelnik JF, et al. Intravitreal aflibercept injection for neovascular age-related macular degeneration: ninety-six-week results of the VIEW studies. Ophthalmology. 2014;121(1):193-201.

12. Boyer DS, Nguyen QD, Brown DM, Basu K, Ehrlich JS; RIDE and RISE Research Group. Outcomes with as-needed ranibizumab after initial monthly therapy: long-term outcomes of the Phase III RIDE and RISE Trials. Ophthalmology. 2015;122(12):2504.e1-2513.e1.

13. Bressler SB, Glassman AR, Almukhtar T, et al. Five-year outcomes of ranibizumab with prompt or deferred laser versus laser or triamcinolone plus deferred ranibizumab for diabetic macular edema. Am J Ophthalmol. 2016;164:57-68.

14. Wykoff CC, Le RT, Khurana RN, et al. Outcomes with as-needed aflibercept and macular laser following the Phase III VISTA DME Trial: ENDURANCE 12-month extension study. Am J Ophthalmol. 2017;173:56-63.

15. Boulanger-Scemama E, Querques G, About F, et al. Ranibizumab for exudative age-related macular degeneration: a five year study of adherence to follow-up in a real-life setting. J Fr Ophtalmol. 2015;38(7):620-627.

16. Mueller S, Agostini H, Ehlken C, Bauer-Steinhusen U, Hasanbasic Z, Wilke T. Patient preferences in the treatment of neovascular age-related macular degeneration: a discrete choice experiment. Ophthalmology. 2016;123(4):876-883.

17. Pauleikhoff D, Bertram B, Holz FG, et al. Die Anti-VEGF-Therapie bei der neovaskulären altersabhängigen Makuladegeneration - therapeutische Strategien: Stellungnahme der Deutschen Ophthalmologischen Gesellschaft, der Retinologischen Gesellschaft und des Berufsverbandes der Augenärzte Deutschlands - November 2014. Der Ophthalmologe. 2015;112(3):237-245. German.

18. Ziemssen F, Helbig H, Lemmen KD, et al. Stellungnahme der Deutschen Ophthalmologischen Gesellschaft, der Retinologischen Gesellschaft und des Berufsverbandes der Augenärzte Deutschlands: Therapie der diabetischen Makulopathie (Stand April 2013). Klinische Monatsblätter für Augenheilkunde. 2013;230(06):614-628. 
19. Fox J. The R commander: a basic statistics graphical user interface to $R$. JSS. 2005;14(9):1-42.

20. Subhi Y, Sørensen TL. Neovascular age-related macular degeneration in the very old ( $\geq 90$ years): epidemiology, adherence to treatment, and comparison of efficacy. J Ophthalmol. 2017;2017:7194927.

21. Storey PP, Murchison AP, Pizzi LT, et al. Impact of physician communication on diabetic eye examination adherence: results from a retrospective cohort analysis. Retina. 2016;36(1):20-27.

22. Sivaprasad S, Hykin P, Chakravarthy U, Lotery A, McKibbin M, Napier J. A retrospective study of the real-life utilization and effectiveness of ranibizumab therapy for neovascular age-related macular degeneration in the UK. Clin Ophthalmol. 2016;10:87-96.
23. Ziemssen F, Eter N, Fauser S, et al. Retrospektive Untersuchung der Anti-VEGF-Behandlungsrealität und Wirksamkeit bei Patienten mit neovaskulärer altersabhängiger Makuladegeneration (nAMD) in Deutschland. [Retrospective investigation of anti-VEGF treatment reality and effectiveness in patients with neovascular age-related macular degeneration (AMD) in Germany: treatment reality of ranibizumab for neovascular AMD in Germany]. Ophthalmologe. 2015;112(3):246-254. German.

\section{Publish your work in this journal}

Clinical Ophthalmology is an international, peer-reviewed journal covering all subspecialties within ophthalmology. Key topics include: Optometry; Visual science; Pharmacology and drug therapy in eye diseases; Basic Sciences; Primary and Secondary eye care; Patient Safety and Quality of Care Improvements. This journal is indexed on

\footnotetext{
Submit your manuscript here: http://www.dovepress.com/clinical-ophthalmology-journal
}

\section{Dovepress}

PubMed Central and CAS, and is the official journal of The Society of Clinical Ophthalmology (SCO). The manuscript management system is completely online and includes a very quick and fair peer-review system, which is all easy to use. Visit http://www.dovepress.com/ testimonials.php to read real quotes from published authors. 